University of Nebraska - Lincoln

DigitalCommons@University of Nebraska - Lincoln

Nutrition and Health Sciences -- Faculty

Publications

Nutrition and Health Sciences, Department of

2019

Milk-Derived Exosomes and Metabolic Regulation

Janos Zempleni

Sonal Sukreet

Fang Zhou

Di Wu

Ezra Mutai

Follow this and additional works at: https://digitalcommons.unl.edu/nutritionfacpub

Part of the Human and Clinical Nutrition Commons, Molecular, Genetic, and Biochemical Nutrition Commons, and the Other Nutrition Commons

This Article is brought to you for free and open access by the Nutrition and Health Sciences, Department of at DigitalCommons@University of Nebraska - Lincoln. It has been accepted for inclusion in Nutrition and Health Sciences -- Faculty Publications by an authorized administrator of DigitalCommons@University of Nebraska Lincoln. 


\title{
Milk-Derived Exosomes and Metabolic Regulation
}

\author{
Janos Zempleni, Sonal Sukreet, Fang Zhou, \\ Di Wu, and Ezra Mutai
}

\footnotetext{
Department of Nutrition and Health Sciences, University of Nebraska-Lincoln, Lincoln, Nebraska 68583-0806, USA

Email: jzempleni2@unl.edu, sonal.sukreet@huskers.unl.edu, fang.zhou@huskers.unl.edu, w19oody@gmail.com, kipronoezr@gmail.com
}

\begin{abstract}
Exosomes are natural nanoparticles that play an important role in cellto-cell communication. Communication is achieved through the transfer of cargos, such as microRNAs, from donor to recipient cells and binding of exosomes to cell surface receptors. Exosomes and their cargos are also obtained from dietary sources, such as milk. Exosome and cell glycoproteins are crucial for intestinal uptake. A large fraction of milk exosomes accumulates in the brain, whereas the tissue distribution of microRNA cargos varies among distinct species of microRNA. The fraction of milk exosomes that escapes absorption elicits changes in microbial communities in the gut. Dietary depletion of exosomes and their cargos causes a loss of circulating microRNAs and elicits phenotypes such as loss of cognitive performance, increase in purine metabolites, loss of fecundity, and changes in the immune response. Milk exosomes meet the definition of bioactive food compounds.
\end{abstract}

Keywords: exosomes, extracellular vesicles, microRNA, milk, RNA

Published in Annual Review of Animal Biosciences 7 (2019), pp 245-62.

DOI: $10.1146020518-115300$

Copyright (C) 2019 by Annual Reviews. Used by permission. 


\section{Endogenous Exosomes}

\section{Biogenesis}

Virtually every living organism, including animals, plants, and microorganisms, releases exosomes and exosome-like vesicles (1-3). Exosomes can be distinguished from other classes of extracellular vesicles (EVs), e.g., microvesicles and apoptotic bodies, by size and biogenesis (4). Exosomes are the smallest EVs known to date and measure approximately $100 \mathrm{~nm}$. Biogenesis of exosomes initiates through the inward budding of vesicles (endocytosis) at the plasma membrane, which leads to the formation of early and late endosomes (Figure 1). Biogenesis continues with the invagination of the late endosomal limiting membrane to form multivesicular bodies and the sorting of cargos into these bodies $(6,7)$. Exosome cargos include various species

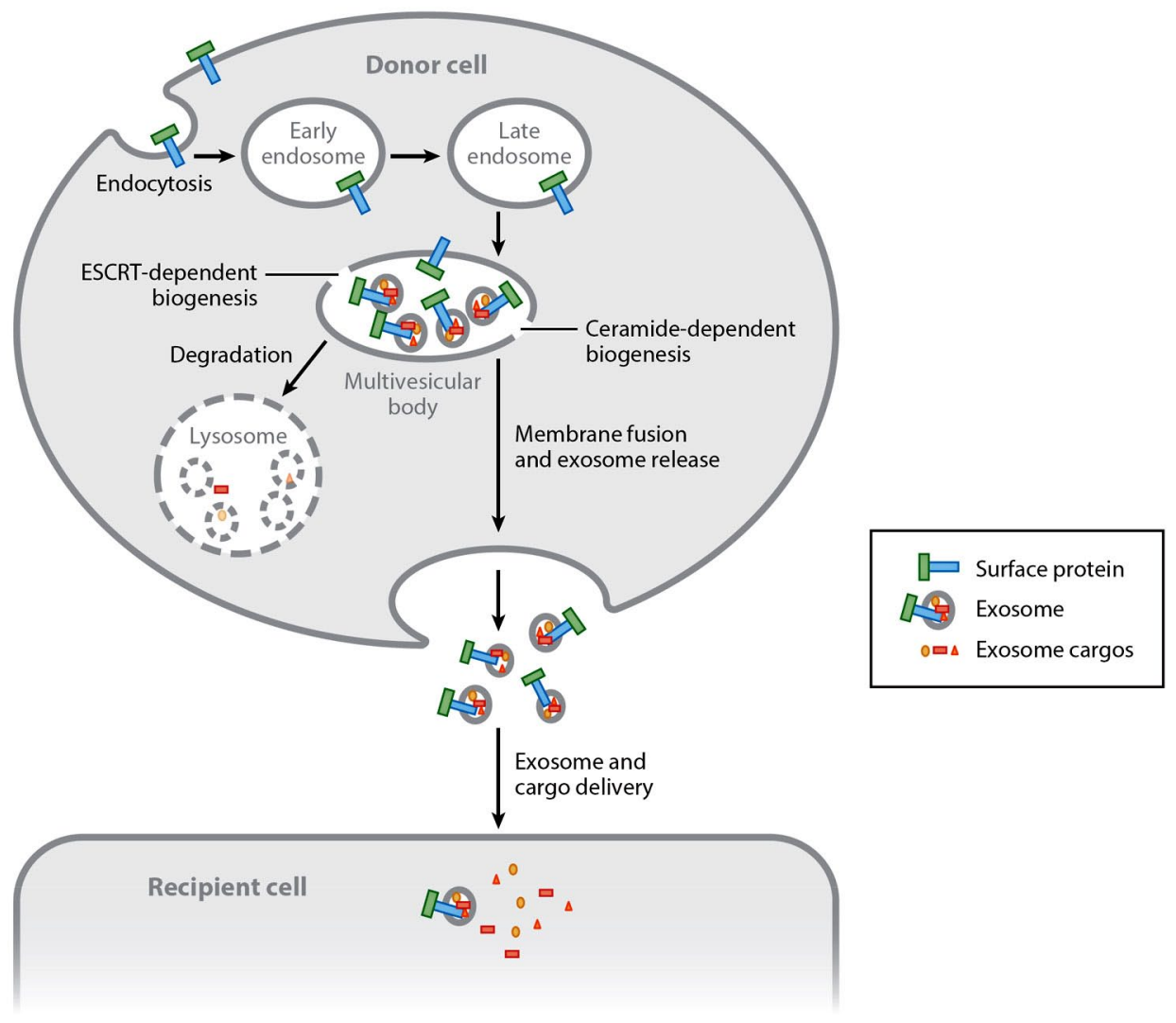

Figure 1 Exosome biogenesis. Figure adapted from Reference 5. Abbreviation: ESCRT, endosomal complex required for transport. 
of coding and noncoding RNAs (ncRNAs), lipids, and proteins (6-9). Exosome biogenesis is achieved through two pathways: the endosomal complex required for transport (ESCRT) and a ceramide-dependent pathway $(10,11)$. Exosomes may be degraded in lysosomes or secreted into the extracellular space upon fusion of multivesicular bodies with the plasma membrane $(6,7)$. Exosomes are present in virtually all body fluids, including milk in humans and animals (12-17). Little is known about the homing signals that direct exosomes to a diverse array of recipient cells. The transmembrane protein CD47 prevents recognition and elimination of exosomes by macrophages, and glycoproteins on the exosome surface play a role in exosome uptake and probably recognition by recipient cells (18-20).

\section{Exosome Cargos}

Exosomes carry diverse cargos, including various species of RNA, proteins, and lipids $(8,21,22)$. The pool of RNA cargos is diverse; e.g., exosomes from human plasma and serum contain ncRNAs such as microRNAs, transfer RNAs (tRNAs), ribosomal RNAs (rRNAs), small nuclear RNAs (snRNAs), piwi-interacting RNAs, and small nucleolar RNAs (snoRNAs) $(8,23,24)$. The profile of ncRNAs in plasma from animals has not been characterized to the same extent as in humans, but microRNAs and piwi-interacting RNAs were detected in bovine plasma (25). That study did not distinguish between exosomal RNAs and RNAs associated with other complexes. A couple of recent papers suggest that RNA contaminants in spin columns might cause artifacts in RNA analyses and offered protocols to decrease the abundance of contaminants $(26,27)$. Exosomes also contain coding RNA, but most of the messenger RNA (mRNA) in exosomes released by human cells is truncated

The distribution patterns of RNA classes and their individual members depend on the tissue in which the exosomes originated; developmental stage; and external factors, such as diet and physical activity (29-32). To date, approximately 1,900 and 800 microRNAs have been reported in humans and cows, respectively (33). RNA loading into exosomes is not a random process; the loading involves sorting mechanisms that favor some cargos over others $(6,7,34)$. For example, the abundance of microRNAs in exosomes secreted by immune cells has a 
pattern distinct from that in the donor cells, and, compared with donor cells, circular RNAs are enriched in the immune cell- derived exosomes $(35,36)$. RNA binding proteins, such as Y-box protein 1, regulate the sorting of distinct microRNAs, such as hsa-miR-223-3p, into human exosomes (37). The proto-oncoprotein KRAS also has been implicated in microRNA sorting to exosomes in colorectal cancer cell lines (38). The binding of RNAs to rafts in the limiting membrane in multivesicular bodies also appears to play a role in the RNA loading process (39). Encapsulation of RNAs in exosomes is of particular importance for the rather labile RNAs because it confers protection against degradation $(36,40)$. To date, 81 and 1,354 proteins have been reported for human and bovine exosomes, respectively $(21,22$; see below). Proteins such as the tetraspanins CD9 and CD63 and the endosomal Alix and Tsg101 are used to authenticate exosome preparations, although the abundance of these markers may vary greatly depending on the donor cell $(41,42)$. Many exosomal proteins are glycosylated, in particular protein domains on the outer surface of the exosomes (43). The mechanisms that regulate protein sorting into exosomes are elusive. Posttranslational modification of proteins, in particular ubiquitination, appears to play a role in the sorting process (44). One interesting approach to studying protein delivery by exosomes included the delivery of ubiquitinated Cre recombinase to exosomes and the delivery of recombinase to various regions of the brain in reporter mice (45).

Little is known about lipid composition and sorting in exosomes. The ExoCarta 2012 database lists approximately 700 exosome lipids, but the data set includes exosomes from sources other than milk (21; see below). The cholesterol and sphingomyelin composition of exosomes from samples other than milk does not depend on donor cells, whereas the content of saturated fatty acids in phosphatidylcholine and phosphatidylethanolamine depends on donor cells (46-48).

Exosomes may carry some DNA. For example, exosomes from cancer patients and cancer cell cultures contain double-stranded DNA, perhaps in the form of chromosomal fragments (49-52). Some exosomal DNA may be of mitochondrial origin (53). Cells might dispose of harmful cytoplasmic DNA through exosome secretion, and investigators have suggested that exosomal DNA might be a biomarker for cancer $(49,52)$. The distribution between DNA inside exosomes and DNA adsorbed to the outer membrane surface is uncertain. 


\section{Cell-to-Cell Communication}

The homing of exosomes probably depends on glycoproteins on the surface of exosomes and recipient cells (20, 54; S. Sukreet \& J. Zempleni, unpublished data). Proteins such as $\mathrm{CD} 47$ on the exosome surface prevent exosome elimination by macrophages $(18,55)$. Although many of the effects of exosome messaging depend on the delivery of exosome cargos to recipient cells, the mere interaction between exosomes and receptor cell surfaces may also elicit cell responses. For example, fibronectin-mediated binding of exosomes to myeloma cells activated $\mathrm{p} 38$ and pERK signaling and expression of downstream target genes $D K K 1$ and $M M P-9$, two molecules that promote myeloma progression (56). Consistent with this theory, exosome internalization was not necessary to elicit gene expression changes in human T cells (57). That said, the vast majority of previous studies in exosome signaling have focused on the delivery of microRNA cargos to the recipient cell interior. MicroRNAs are approximately 20-24 nucleotides long, and sequence complementarity in the seed region (nucleotides 2-8) is of particular importance for the binding of microRNAs to their mRNA targets $(58,59)$. Presumably, the bias toward studies of microRNA cargos is due to the fact that more than $60 \%$ of human genes are putative targets for regulation by microRNAs, as well as the large number of microRNAs identified in humans and animals $(33,60)$. MicroRNAs decrease the expression of target genes by binding to complementary sequences primarily in the $3^{\times}$untranslated region of mRNAs (Figure 2), although some microRNAs may also bind to sequences in the $5^{\times}$untranslated region and the coding sequence $(58,59,61)$. Binding sites in the $5^{\times}$ untranslated region and coding sequence in mRNAs are rare, but they exist (62). Binding is facilitated by argonaute proteins and leads to the formation of a ribonucleoprotein complex referred to as RNAinduced silencing complex (RISC); RISC is a $5^{\times}$phosphomonoesterproducing endonuclease (63). If the sequence complementarity between microRNA and its mRNA target is high, the mRNA is degraded $(58,59,63)$. If the complementarity outside the seed region is low, the mRNA in the microRNA/mRNA duplex is not translated. The absence of degradation is due to poor positioning of the mRNA toward the catalytic domain in RISC $(58,59)$. 


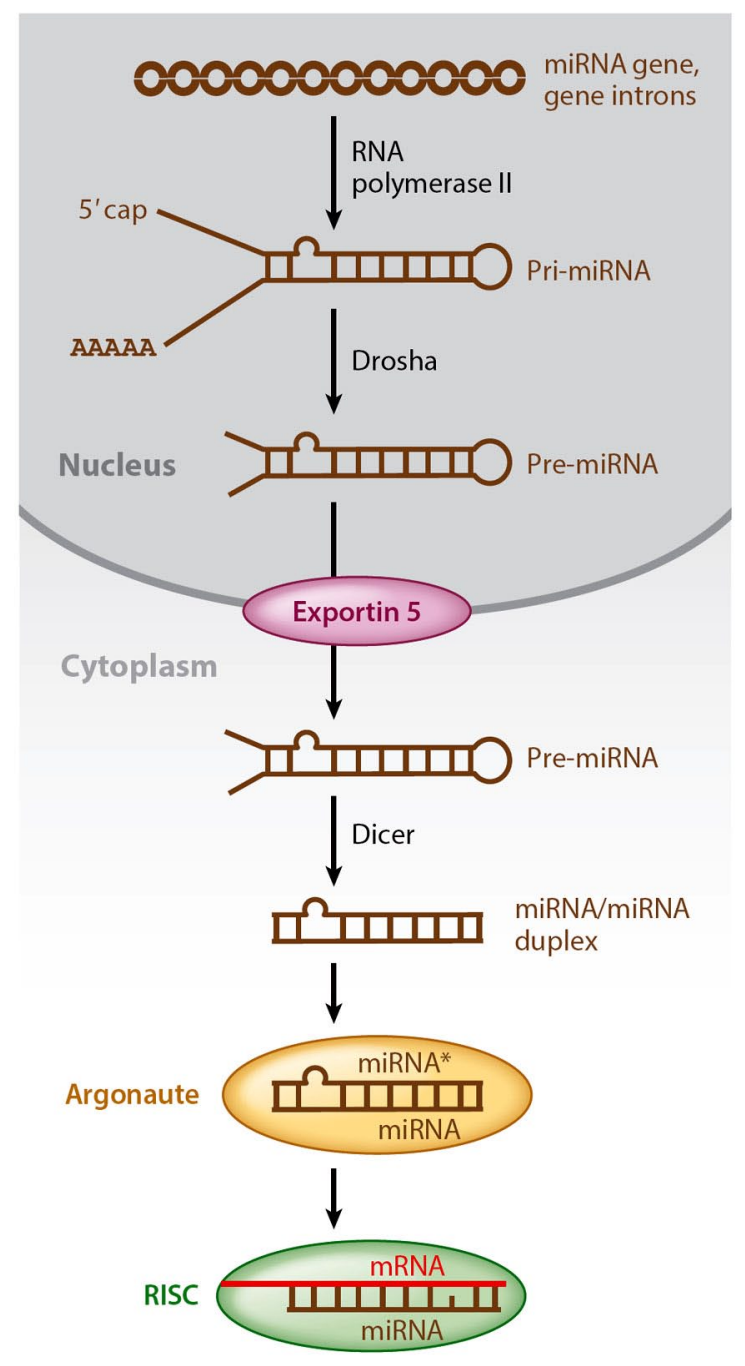

Figure 2 Gene repression by microRNAs. Figure adapted from Reference 5. Abbreviations: miRNA, microRNA; mRNA, messenger RNA; RISC, RNA-induced silencing complex.

Noncanonical pathways of microRNA signaling, such as binding to Toll-like receptors (TLRs), are of particular interest because of the roles of TLRs in host defense mechanisms (64). TLRs are widely appreciated for their ability to bind single-stranded (TLR3, TLR7, TLR8) and double-stranded RNA (TLR3) as part of antiviral response mechanisms (65-75). Single-stranded RNAs require endosomal delivery (exosomes) for stabilization against ribonucleases and activation of TLRs (76). TLRs reside on the cell surface or in the cell interior and may 
Table 1 Toll-like receptors and their cellular localization and ligands (65-75)

\begin{tabular}{lll} 
TLR & Localization & Ligand(s) \\
\hline 1 & Cell surface & Triacyl glycopeptides \\
2 & Cell surface & Numerous \\
3 & Cell compartment & ss/dsRNA \\
4 & Cell surface & Diverse \\
5 & Cell surface & Flagellin, profilin \\
6 & Cell surface & Multiple diacyl lipopeptides \\
7 & Cell compartment & ssRNA and others \\
8 & Cell compartment & ss viral RNA \\
9 & Cell compartment & Unmethylated CpG deoxynucleotide DNA \\
10 & Unknown & Unknown \\
11 & Cell compartment & Profilin \\
12 & Unknown & Profilin \\
13 & Cell compartment & Bacterial rRNA (CGGAAAGACC) \\
\hline
\end{tabular}

Abbreviations: ds, double-stranded; rRNA, ribosomal RNA; ss, single-stranded; TLR, Tolllike receptor.

be able to amplify weak microRNA signals (Table 1). Note that RNA other than microRNA in exosomes might also bind to TLRs, although this possibility has not yet been explored. The relevance of mRNA ligands in TLR activation and antiviral response is underscored by the observations that self-amplifying mRNA vaccines confer protection against influenza viruses, and bacterial RNAs stimulate innate immune responses $(77,78)$.

Prokaryotic and eukaryotic microbes communicate with their environment through EVs, suggesting that gut microbes might interact with their host through EVs (3). The communication between microbes and environment includes Gram-positive bacteria, which use EVs to communicate despite having to transport EVs across their cell walls (79, 80). Exosomes secreted by Leishmania deliver proteins to macrophages in the host, and infection-like stressors upregulate exosome secretion by Leishmania (81). Viruses may participate in exosome signaling through hi-jacking and modifying exosomes (82). 


\section{Bioavailability and distribution of milk exosomes and their cargos}

\section{Exosomes}

In 2012, evidence emerged that MIR168a from dietary sources (rice) is bioavailable across species boundaries (83). This discovery has sparked great interest in the bioavailability and distribution of milk exosomes and their cargos. The findings from these studies are discussed in the sections below.

Bioavailability and intestinal transport. The paradigm that exosomes and their cargos are exclusively derived from endogenous synthesis has been challenged in a series of studies illustrating that exosomes and their RNA cargos in bovine milk are bioavailable in humans and mice, i.e., across species boundaries (14, 84, 85). These observations were corroborated in cell culture studies, which suggested that Caco-2 human intestinal cells, IEC-6 primary rat small intestinal cells, human umbilical cord vascular endothelial cells, and THP-1 human macrophage-like cells take up bovine milk exosomes (86-88). The uptake of milk exosomes by endocytosis follows Michaelis-Menten kinetics in Caco-2 and IEC-6 cells and venous endothelial cells $(86,88)$. Protease treatment and excess glucose and galactose inhibited the uptake of milk exosomes in intestinal cell cultures, suggesting that exosome recognition depends on glycoproteins on the surface of exosomes and cells $(86,88)$. Sukreet and colleagues identified 90 and 41 glycan features in protein loops exposed on the outer surface of bovine milk exosomes and Caco-2 cells (Table 2) (89; S. Sukreet \& J. Zempleni, unpublished data). Enzymatic removal of glycans on the surface of milk exosomes and human intestinal Caco-2 and human primary fetal small intestinal (FHs) cells resulted in a significant loss in the uptake of fluorophore (FM 4-64)-labeled exosomes by the respective target cells (S. Sukreet \& J. Zempleni, unpublished data). $N$-acetylglucosamine and $\alpha$-Nacetylgalactosamine modifications were particularly important for the uptake of bovine milk exosomes by Caco-2 and FHs cells. The apparent bioavailability of bovine milk exosomes was estimated to be $5 \%$ in mice, but that estimate is based on using a lipophilic fluorophore label (1,1-dioctadecyl-3,3,3,3-tetramethylindotricarbocyanine iodide, DiR) known to transfer from exosomes to 
Table 2 Pool of total proteins and glycoproteins on the surface of bovine milk exosomes and Caco-2 cells ${ }^{a}$

\begin{tabular}{lcc} 
Proteins & $\begin{array}{c}\text { Number of proteins } \\
\text { Exosomes }\end{array}$ & Caco-2 cells \\
\hline Total proteins & 484 & 929 \\
Glycoproteins on the outer surface & 90 & 41 \\
Glycan modifications & 55 & 29 \\
N-Glycans & 19 & 6 \\
O-Glycans & 16 & 6 \\
C-Glycans & & \\
\hline
\end{tabular}

a. Proteins were identified by liquid chromatography-mass spectrometry/mass spectrometry with (exosomes) and without (Caco-2 cells) prior enzymatic removal of glycan features. Glycan modifications impair the detection of proteins; i.e., the number of proteins reported for Caco-2 cells is probably an underestimate. The localization of proteins to membranes and the orientation of glycans were predicted by using TMHMM, NetCGlyc, NetNGlyc, and NetOGlyc (90-93).

proteins and lipoproteins and presumably other structures (94). Moreover, estimates of exosome bioavailability based on DiR-labeled exosomes are approximately five times lower than estimates based on using fluorophore-tagged microRNA cargos, and the apparent distribution of DiR-labeled milk exosomes is different from that of any other exosome label tested (95). Enzymatic removal of N-glycans on the surface of bovine milk exosomes prior to delivery by oral gavage resulted in a significant loss in exosome bioavailability in mice (S. Sukreet \& J. Zempleni, unpublished data). The apparent bioavailability of milk exosomes is higher if exosomes from the same species are ingested compared with administration across species boundaries. For example, visual inspection of images in which endogenously labeled exosomes were delivered through the natural route of suckling in mice appears to suggest a bioavailability of more than $25 \%$, although the authors did not attempt to quantify the bioavailability of murine milk exosomes in mouse pups (95).

Distribution. DiR-labeled bovine milk exosomes, administered through oral gavage, accumulated in liver, spleen, pancreas, and kidney but were also detectable in lung, colon, and brain in nude mice (96). These studies used doses of exosomes far higher than what can 
be achieved through dietary intake, lacked important controls, and disregarded the differences in distribution of the DiR label discussed above. Subsequent studies with lower concentrations and additional controls suggest that the majority of DiR-labeled bovine milk exosomes accumulate in the intestinal mucosa, liver, and large intestinal content, and concentrations in other tissues were barely higher than background (95). Presumably, accumulation of milk exosomes in liver and spleen depends on resident macrophages in these tissues (97). Consistent with the theory of macrophage-dependent distribution of milk exosomes, depletion of macrophages by treating mice with clodronate resulted in an almost exclusive accumulation of exosomes in the liver and a loss of accumulation in the spleen after oral gavage (95). Likewise, a loss of exosome accumulation in the liver was observed when proteins were removed from the surface of exosomes with trypsin prior to oral gavage. Glycoproteins on the surface of DiR-labeled exosomes are important for recognition by target cells (including macrophages), because enzymatic removal of N-glycans caused a loss of milk exosomes delivered by oral gavage in mice (S. Sukreet \& J. Zempleni, unpublished data). Bovine milk exosomes, administered by oral gavage, accumulated in the placenta and were transferred to fetuses in C57BL/6 mice (M. Sadri \& J. Zempleni, unpublished data). Notwithstanding concerns regarding distribution artifacts caused by DiR, these initial studies provided important first insights on milk exosome distribution. Subsequent studies assessed the distribution of milk exosomes and their RNA cargos by using labels other than DiR.

The distribution of exosomes in porcine milk, endogenously labeled with a fluorescent protein (ZsGreen1), was different from that observed for DiR-labeled bovine milk exosomes. Porcine milk exosomes accumulated in the liver and brain after intravenous injection in mice and in the brain in wild-type piglets nursed by ZsGreen1 sows (95). A similar pattern of exosome distribution was observed when wild-type mouse pups were nursed by transgenic dams expressing a fluorescent fusion protein in milk; exosomes were detected in the heart, lungs, kidneys, liver, and brain of pups. The distribution patterns of exosomes labeled with fluorescent proteins probably are more accurate than the patterns of DiR-labeled exosomes, because DiR may detach from exosomes and transfer to protein and lipoproteins ( 94 ; see below). 


\section{MicroRNAs}

MicroRNAs are highly conserved among species and play important roles in gene regulation (see above). Studies in plant foods provided evidence that dietary microRNAs are bioavailable across species boundaries (83). To date, the majority of studies assessing the bioavailability of cargos in milk exosomes have focused on microRNAs.

Bioavailability and intestinal transport. Various studies identified between 100 and 1,500 species of microRNAs in bovine milk exosomes (87, 98-101; S. Sukreet \& J. Zempleni, unpublished data). The variation among these studies is due to differences in experimental conditions, including the stringency applied in bioinformatics analysis (mismatches allowed), use of raw milk versus processed milk, choice of sample (milk versus whey fraction versus exosomes), inclusion versus exclusion of novel microRNAs, and platforms used [microarray versus RNA-sequencing (RNA-Seq) analysis]. Although bovine milk is the most heavily investigated species, data are also available for humans, pigs, giant pandas, and goats $(16,17,102,103)$. Encapsulation of microRNAs in exosomes confers protection against degradation by harsh conditions such as low pH and RNases, as encountered in the gastrointestinal tract $(99,104)$. Some loss of microRNA occurs during the processing of raw milk and through the microwaving of milk (105).

Evidence suggests that milk microRNAs enter recipient cells through the endocytosis of their exosome shells $(86,88)$. Data from studies in dual-chamber systems suggest that some microRNAs cross the intestinal mucosa more efficiently than others, and reverse transport from the basolateral to the luminal side is minimal in human Caco-2 colon carcinoma cells (86). It remains to be determined whether the discrimination among distinct species of microRNAs in the transport across the intestinal mucosa also depends on Y-box protein 1 and KRAS, as described above, or whether endogenous mRNAs act as microRNA sponges, leading to RNA degradation $(37,38,106)$. As an alternative to exosome-dependent transport of microRNAs, a transporter for plant-derived microRNAs is expressed in the stomach and to a lesser extent in spleen, lung, liver, kidney, heart, brain, and skeletal muscle in mice. The identity of the transporter is privileged information, and readers are referred to an upcoming publication (C.Y. Zhang, Nanjing University, personal communication). 
Evidence is accumulating that microRNAs in bovine and porcine milk are bioavailable in humans, pigs, and mice $(14,84,85,107$; reviewed in 5). MicroRNAs in bovine milk enter circulating immune cells in humans and cell cultures and elicit changes in gene expression (14, 99). Moreover, microRNAs may bind to Toll-like receptors $(64,108)$. Numerous independent laboratories have contributed evidence in support of the theory that dietary microRNAs, in addition to those from milk, are bioavailable across species boundaries (84, 86-88, 96, 108120). That said, there is also evidence that postprandial increases of some microRNAs in plasma may be due to a food-induced increase in endogenous microRNA synthesis in humans (121).

In the spirit of transparency, we briefly discuss concerns raised regarding the bioavailability of microRNAs in milk in papers by Laubier et al. (122), Auerbach et al. (123), Title et al. (124), and Kang et al. (125). Laubier et al. (122) fostered wild-type pups to transgenic mice that overexpressed miR-3ob and failed to see a substantial increase in tissue levels of miR-3ob in pups. The failure to observe an increase in miR-3ob in pup tissues was probably due to the fact that the miR$30 \mathrm{~b}$ in overexpression dams was not encapsulated in milk exosomes, thereby compromising miR-3ob stability and bioavailability (5, 86, 99, 104). Auerbach et al. (123) reported a failure to detect bovine miR29b and miR-200c in human plasma following consumption of milk. Subsequent studies suggest that the integrity of the samples used in that study was compromised and the RNA was degraded (85). Title et al. (124) detected only trace amounts of miR-375 in the plasma of miR-375 knockout mouse pups fostered to wild-type dams. Our studies suggest that unlike many other microRNAs, miR-375 in milk is subject to "first passage elimination" in intestinal mucosa and liver, and, therefore, its concentrations in circulation and peripheral tissues are low $(95,126,127)$. Kang et al. (125) conducted a meta-analysis of published RNA-Seq data sets and concluded that the abundance of dietary microRNAs in body fluids is very low and possibly due to assay artifacts. Their analysis is biased by applying considerably lower levels of stringency when mapping human microRNAs compared with dietary microRNAs, by disregarding the abundance of microRNAs in foods, by withholding details of data normalization protocols across data sets, and by dismissing the possibility that local concentrations of dietary microRNAs at the site of absorption might be high. This being said, sample contamination in RNA-Seq analysis through reagents 
is a potential pitfall in microRNA analysis and needs to be taken into account during sample preparation (26). Voices of caution deserve recognition despite their unfortunate focus on how many copies of microRNAs are delivered to cells $(124,128)$. The binding of microRNAs to mRNAs does not elicit microRNA decay when sequence complementarity is low; in such cases, low copy numbers of microRNAs may result in the degradation of a large number of mRNA copies, analogous to enzyme-dependent reactions $(129,130)$. It appears to be more productive to answer the question of how milk microRNAs elicit changes in gene expression and phenotypes than to engage in discussions about how many copies of microRNAs are absorbed and delivered to cells $(131,132)$.

Distribution. Distinct species of synthetic fluorophore-labeled microRNAs, transfected into bovine milk exosomes and administered by oral gavage, have unique distribution profiles in mice (95). For example, miR-375 accumulates primarily in the intestinal mucosa, liver, and brain (with some accumulation in kidneys); miR-320a accumulates primarily in the liver; miR-155-5p accumulates primarily in the spleen; and miR-34a accumulates primarily in the brain. The authors used a dual fluorophore and quencher strategy to demonstrate that the fluorophore label was not released from microRNAs following administration by oral gavage; i.e., microRNAs (as opposed to released label) were traced.

\section{RNAs Other Than MicroRNAs}

The following species of RNA were identified in bovine milk exosomes, in order of abundance: tRNAs, but also some rRNAs, snRNAs, snoRNAs, repetitive sequences, and nonannotated sequences (100). snRNAs play a role in the splicing of premessenger RNA in the spliceosome (133), whereas snoRNAs primarily guide modifications of rRNA, tRNA, and snRNA (134). The functions of these ncRNAs in milk are unknown. Studies that focused on the discovery of coding RNA in milk exosomes identified approximately 19,000 and 3,200 bovine mRNAs by microarray and RNA-Seq analysis, respectively (87; S. Sukreet \& J. Zempleni, unpublished data). The vast majority of mRNAs were truncated with a bias toward the $3^{\times}$end of the mRNAs (S. Sukreet \& J. Zempleni, unpublished data). Approximately 100 of the truncated 
transcripts retained a natural AUG start codon (D. Wu \& J. Zempleni, unpublished data). The mRNAs in milk exosomes might be translated into protein using in vitro translation protocols and U937 cells, suggesting that foreign proteins might originate from milk exosomes in host species (D. Wu \& J. Zempleni, unpublished data). These observations implicate milk exosomes in food allergies and immune tolerance, although this possibility remains to be tested.

Of the non-bovine mRNAs in bovine milk exosomes, $83 \%$ and $38 \%$ mapped to microbial species in raw milk and store-bought milk, respectively. Studies using fluorophore-labeled synthetic fragments of microbial mRNAs suggest that the mRNAs enter cells in the intestinal mucosa (D. Wu \& J. Zempleni, unpublished data). Synthetic microbial mRNA, transfected into liposomes, activated interferon- $\beta$ in reporter cell cultures (F. Zhou \& J. Zempleni, unpublished data).

\section{Interactions of Milk Exosomes with the Gut Microbiome}

A physiologically significant fraction ( $75 \%$ ) of foreign milk exosomes escapes absorption and enters the large intestine (95). As described above, microorganisms communicate with their environment through EVs. Consistent with this premise, consumption of an exosome- and RNA-depleted (ERD) diet elicited changes in microbial communities in mice compared with mice fed an exosome- and RNA-sufficient (ERS) control diet (see the sidebar titled Exosome- and RNA-Defined Diets). An ERD diet caused a change in the prevalence of bacteria in 5 microbial phyla, 7 orders, and 52 operational taxonomic units compared with controls fed an ERS diet at ages 7, 15, and 47 weeks (F. Zhou \& J. Zempleni, unpublished data).

There is a precedent for milk constituents conferring a growth advantage to select gut microbes. For example, human milk oligosaccharides selectively amplify populations of Bifidobacterium infantis (136). The effects of milk exosome-induced changes in microbial communities on host phenotypes remain to be assessed. A recent report suggests that the selection pressure of milk exosomes is sufficiently strong to cause an enrichment of polymorphisms and mutations of murine rectal bacteria in both exosome-free and exosome-supplemented cultures compared with reference genomes (F. Zhou \& J. Zempleni, unpublished data). 


\section{Exosome- and RNA-defined diets}

Exosome- and RNA-depleted (ERD) and exosome- and RNAsufficient (ERS) diets are based on an AIN-93G formulation and were referred to as ExoMinus and ExoPlus diets in the original publication $(14,135)$. In these diets, lyophilized milk powder (and soy protein) substitutes for milk casein in the AIN-93G formula to prepare a diet lacking dairy exosomes in the casein fraction. Macronutrients and micronutrients other than exosomes and their RNA cargos are the same in ERD and ERS diets. The milk added to the diets provides the equivalent of $0.5 \mathrm{~L}$ of milk consumed by a human adult per mouse and day. The milk used to prepare the powder for the ERD diet is ultrasonicated for $1.5 \mathrm{~h}$ and incubated for $1 \mathrm{~h}$ at $37^{\circ} \mathrm{C}$ prior to lyophilization; the milk used to prepare the powder for the ERS is not ultrasonicated. Ultrasonication leads to a transient disruption of exosome membranes and depletion of RNA cargos in exosomes. Exosome membranes close during incubation, i.e., the number of exosomes remains the same in ERD and ERS milk. In the initial paper reporting the use of ERD and ERS diets, microRNA depletion was confirmed by quantitative real-time PCR analysis of miR-29b and miR-200c. Subsequent studies confirmed and expanded these finding by using RNA-sequencing (RNA-Seq) analysis, suggesting a near-complete loss of both microRNAs and messenger RNAs in ERD milk (S. Sukreet \& J. Zempleni, unpublished data). Recent evidence suggests that ultrasonication causes not only a depletion of RNA cargos but also a decrease in the transport of exosomes by intestinal cells (S. Sukreet \& J. Zempleni, unpublished data). Presumably, the decrease in cellular uptake of exosomes is due to sonication-induced changes in exosome morphology and lipid composition of the exosome membrane bilayer. Changes in surface glycoprotein features might also contribute to the loss in bioavailability. That said, the loss of RNA cargos in combination with poor bioavailability of sonicated exosomes makes ERD and ERS diets a powerful tool to assess phenotypes of dietary milk exosome and RNA depletion. Note that plasma levels of miR$29 \mathrm{~b}$ and miR-200c decreased by approximately 60\% in C57BL/6 mice fed the ERD diet for four weeks, starting at age three weeks, compared with ERS controls (14). 


\section{Phenotypes of dietary depletion of exosomes and cargos}

ERD and ERS diets were used to assess the phenotypes from dietary depletion of bovine milk exosomes in mice. Effects on brain function were among the strongest phenotypes observed. For example, female C57BL/ 6 mice fed the ERD diet displayed a loss of spatial learning and memory (SLM) compared with ERS controls (137). Effects of diet were more pronounced in pups born to parents fed the ERD diet and continued on the parental diets compared with mice started on experimental diets at age three weeks. Effects of the diets on SLM were similar in pups ages four and seven weeks. Aberrant metabolism of purines has been implicated in the loss of SLM in mice fed the ERD diet. The purine metabolites adenosine and ATP play an important role in SLM, and hepatic levels of purine metabolites were substantially higher in mice fed the ERD diet compared with ERS controls $(138,139)$. Effects of milk feeding on purine metabolites were similar in human cohorts compared with the changes observed in mice: The concentrations of purine metabolites were higher in plasma and urine in human milk avoiders compared with milk consumers and in infants fed soy formula compared with infants fed human milk or milk formula (139). The mechanisms by which milk exosomes and RNA cargos alter purine metabolism is unknown, although the authors proposed that miR362-3p and miR-30a-5p in milk exosomes might be responsible for the differential expression of $5^{\times}$-nucleotidase, cytosolic IIIB, and adenosine deaminase in livers from mice fed ERD or ERS diets. Effects of milk exosome intake on brain biology were corroborated in studies of kainic acid-induced seizures (E. Mutai \& J. Zempleni, unpublished data). Seizure severity was stronger in mice fed an ERD diet than in ERS controls. Note that food and water intake, body composition, physical activity, and protein metabolism were the same in mice fed ERD and ERS diets (140).

Dietary depletion of milk exosomes and RNA cargos caused a loss in fecundity. When male and female mice fed ERD were mated in all possible permutations [male ERD $\times$ female ERD, male ERS $\times$ female ERD, male ERD $\times$ female ERS, and male ERS $\times$ female ERS (control)], the litter size produced by ERS/ERS breeders was twice that of the litter size in other groups (M. Sadri \& J. Zempleni, unpublished data). Seventeen genes were differentially expressed in placentas from ERD 
and ERS dams; the majority of these genes are implicated in cell adhesion, suggesting that the decrease in litter sizes might have been due to loss of implantation. This theory is consistent with previous observations that (endogenous) miR-3od is crucial for fetal implantation, and synthetic miR-3od, transfected into bovine milk exosomes and delivered orally to pregnant mice on gestational day 16.5 , accumulates in the placenta and fetus $(141,142)$.

Exosome- and RNA-defined diets elicited moderate changes in immune responses at the intestinal level. Histopathology analysis suggested that the composite score of inflammatory bowel disease was lower in the cecum of male $\mathrm{Mdr}^{-/-}$mice fed ERS compared with male Mdr1a ${ }^{-/-}$mice fed ERD (D. Wu \& J. Zempleni, unpublished data). $\mathrm{Mdr}_{\mathrm{a}} \mathrm{a}^{-/-}$mice are genetically predisposed to developing inflammatory bowel disease (143). The proinflammatory effect of ERD was attributed to an increased expression of the proinflammatory chemokine MIG in ERD mice compared with ERS controls. Eighty-seven genes were differentially expressed in the cecum of the dietary groups in $\mathrm{Mdr1a}^{-/-}$mice; 16 of the differentially expressed genes are implicated in immune function and inflammation.

Milk exosomes and their microRNA cargos elicit a modest, if any, response from the human immune system. For example, the secretion of proinflammatory (IL-1 $\beta$, IL- 6 , and TNF- $\alpha$ ) and anti-inflammatory (IL-10) cytokines by human peripheral blood mononuclear cells (PBMCs) ex vivo was not affected by milk consumption prior to PBMC collection or by the addition of bovine milk exosomes transfected with four immune-relevant microRNAs (miR-15b, miR-21, miR155, and miR-223) to culture media. Milk exosomes and their cargos also elicited an immune response in healthy humans (E. Mutai \& J. Zempleni, unpublished data). These observations are consistent with a moderate, nonsignificant increase in plasma cytokine concentrations following oral administration of bovine milk in nude mice (144).

\section{Conclusions and future outlook}

The field of dietary exosomes and RNAs, particularly those in milk, has witnessed rapid expansion and progress during the past four years. But much uncharted territory remains to be explored. Examples include the discovery of the mechanisms through which dietary 
depletion of milk exosomes elicits phenotypes, exosome-microbiome interactions, and the importance of exosomes and their cargos in human milk and infant formulas in infant nutrition. The use of milk exosomes in drug delivery also is a potentially rewarding field, which would benefit greatly from the development of protocols to direct drug-loaded exosomes to specific tissues.

Disclosure J. Zempleni serves as a consultant for PureTech Health, Inc.

Acknowledgments This work was supported by the National Institute of Food and Agriculture (NIFA), US Department of Agriculture (USDA), under award number 2015-67017-23181; National Institutes of Health (NIH) grants 1P2OGM104320 and NIFA2016-67001-25301; PureTech Health, Inc.; the Gerber Foundation; the Egg Nutrition Center; the University of Nebraska Agricultural Research Division (Hatch Act); and USDA multistate group W4002 (all to J.Z.).

\section{Literature cited}

1. Valadi H, Ekstrom K, Bossios A, Sjostrand M, Lee JJ, Lotvall JO. 2007. Exosomemediated transfer of mRNAs and microRNAs is a novel mechanism of genetic exchange between cells. Nat. Cell Biol. 9:654-59

2. Hansen LL, Nielsen ME. 2017. Plant exosomes: Using an unconventional exit to prevent pathogen entry? J. Exp. Bot. 69:59-68

3. Wolf JM, Casadevall A. 2014. Challenges posed by extracellular vesicles from eukaryotic microbes. Curr. Opin. Microbiol. 22C:73-78

4. Gyo“ rgy B, Szabo' TG, Pa'szto'i M, Pa'1 Z, Misja'k P, et al. 2011. Membrane vesicles, current state-of-the-art: emerging role of extracellular vesicles. Cell. Mol. Life Sci. 68:2667-88

5. Zempleni J, Aguilar-Lozano A, Sadri M, Sukreet S, Manca S, et al. 2017. Biological activities of extracellular vesicles and their cargos from bovine and human milk in humans and implications for infants. J. Nutr. 147:3-10

6. Yanez-Mo M, Siljander PR, Andreu Z, Zavec AB, Borras FE, et al. 2015. Biological properties of extracellular vesicles and their physiological functions. J. Extracell. Vesicles 4:27066

7. Abels ER, Breakefield XO. 2016. Introduction to extracellular vesicles: biogenesis, RNA cargo selection, content, release, and uptake. Cell. Mol. Neurobiol. 36:301-12

8. Huang X, Yuan T, Tschannen M, Sun Z, Jacob H, et al. 2013. Characterization of human plasma-derived exosomal RNAs by deep sequencing. BMC Genom. 14:319

9. Raposo G, Stoorvogel W. 2013. Extracellular vesicles: exosomes, microvesicles, and friends. J. Cell Biol. 200:373-83 
10. Hurley JH. 2008. ESCRT complexes and the biogenesis of multivesicular bodies. Curr. Opin. Cell Biol. 20:4-11

11. Trajkovic K, Hsu C, Chiantia S, Rajendran L, Wenzel D, et al. 2008. Ceramide triggers budding of exosome vesicles into multivesicular endosomes. Science 319:1244-47

12. La"sser C, Alikhani VS, Ekstro“ m K, Eldh M, Paredes PT, et al. 2011. Human saliva, plasma and breast milk exosomes contain RNA: uptake by macrophages. J. Transl. Med. 9:9

13. Gu Y, Li M, Wang T, Liang Y, Zhong Z, et al. 2012. Lactation-related microRNA expression profiles of porcine breast milk exosomes. PLOS ONE 7:e43691

14. Baier SR, Nguyen C, Xie F, Wood JR, Zempleni J. 2014. MicroRNAs are absorbed in biologically meaningful amounts from nutritionally relevant doses of cow's milk and affect gene expression in peripheral blood mononuclear cells, HEK293 kidney cell cultures, and mouse livers. J. Nutr. 144:1495-500

15. Yassin AM, Hamid MIA, Farid OA, Amer H, Warda M. 2016. Dromedary milk exosomes as mammary transcriptome nano-vehicle: their isolation, vesicular and phospholipidomic characterizations. J. Adv. Res. 7:749-56

16. Ma J, Wang C, Long K, Zhang H, Zhang J, et al. 2017. Exosomal microRNAs in giant panda (Ailuropoda melanoleuca) breast milk: potential maternal regulators for the development of newborn cubs. Sci. Rep. 7:3507

17. Golan-Gerstl R, Elbaum Shiff Y, Moshayoff V, Schecter D, Leshkowitz D, Reif S. 2017. Characterization and biological function of milk-derived miRNAs. Mol. Nutr. Food Res. 61:1700009

18. Chauhan S, Danielson S, Clements V, Edwards N, Ostrand-Rosenberg S, Fenselau C. 2017. Surface glycoproteins of exosomes shed by myeloid-derived suppressor cells contribute to function. J. Proteome Res. 16:238-46

19. Liu X, Pu Y, Cron K, Deng L, Kline J, et al. 2015. CD47 blockade triggers T cellmediated destruction of immunogenic tumors. Nat. Med. 21:1209-15

20. Escrevente C, Keller S, Altevogt P, Costa J. 2011. Interaction and uptake of exosomes by ovarian cancer cells. BMC Cancer 11:108

21. Mathivanan S, Fahner CJ, Reid GE, Simpson RJ. 2012. ExoCarta 2012: database of exosomal proteins, RNA and lipids. Nucleic Acids Res. 40:D1241-D44

22. Kalra H, Simpson RJ, Ji H, Aikawa E, Altevogt P, et al. 2012. Vesiclepedia: a compendium for extracellular vesicles with continuous community annotation. PLOS Biol. 12:e1001450

23. Li M, Zeringer E, Barta T, Schageman J, Cheng A, Vlassov AV. 2014. Analysis of the RNA content of the exosomes derived from blood serum and urine and its potential as biomarkers. Philos. Trans. R. Soc. Lond. B Biol. Sci. 369:20130502

24. Freedman JE, Gerstein M, Mick E, Rozowsky J, Levy D, et al. 2016. Diverse human extracellular RNAs are widely detected in human plasma. Nat.

Commun. 7:11106

25. Spornraft M, Kirchner B, Haase B, Benes V, Pfaffl MW, Riedmaier I. 2014. Optimization of extraction of circulating RNAs from plasma-enabling small RNA sequencing. PLOS ONE 9:e107259 
26. Heintz-Buschart A, Yusuf D, Kaysen A, Etheridge A, Fritz JV, et al. 2018. Small RNA profiling of low biomass samples: identification and removal of contaminants. BMC Biol. 16:52

27. Habier J, May P, Heintz-Buschart A, Ghosal A, Wienecke-Baldacchino AK, et al. 2018. Extraction and analysis of RNA isolated from pure bacteria-derived outer membrane vesicles. Methods Mol. Biol. 1737:213-30

28. Batagov AO, Kurochkin IV. 2013. Exosomes secreted by human cells transport largely mRNA fragments that are enriched in the $3^{\times}$-untranslated regions.

Biol. Direct 8:12

29. Vallabhajosyula P, Korutla L, Habertheuer A, Yu M, Rostami S, et al. 2017. Tissue-specific exosome biomarkers for noninvasively monitoring immunologic rejection of transplanted tissue. J. Clin. Investig. 127:1375-91

30. Fernandez-Valverde SL, Taft RJ, Mattick JS. 2010. Dynamic isomiR regulation in Drosophila development. RNA 16:1881-88

31. Tarallo S, Pardini B, Mancuso G, Rosa F, Di Gaetano C, et al. 2014. MicroRNA expression in relation to different dietary habits: a comparison in stool and plasma samples. Mutagenesis 29:385-91

32. Aoi W, Ichikawa H, Mune K, Tanimura Y, Mizushima K, et al. 2013. Muscleenriched microRNA miR-486 decreases in circulation in response to exercise in young men. Front. Physiol. 4:80

33. Kozomara A, Griffiths-Jones S. 2014. miRBase: annotating high confidence microRNAs using deep sequencing data. Nucleic Acids Res. 42:D68-D73

34. Stevanato L, Thanabalasundaram L, Vysokov N, Sinden JD. 2016. Investigation of content, stoichiometry and transfer of miRNA from human neural stem cell line derived exosomes. PLOS ONE 11:eo146353

35. Squadrito ML, Baer C, Burdet F, Maderna C, Gilfillan GD, et al. 2014. Endogenous RNAs modulate microRNA sorting to exosomes and transfer to acceptor cells. Cell Rep. 8:1432-46

36. Li Y, Zheng Q, Bao C, Li S, Guo W, et al. 2015. Circular RNA is enriched and stable in exosomes: a promising biomarker for cancer diagnosis. Cell Res. 25:981-84

37. Shurtleff MJ, Temoche-Diaz MM, Karfilis KV, Ri S, Schekman R. 2016. Y-box protein 1 is required to sort microRNAs into exosomes in cells and in a cellfree reaction. eLife 5:e19276

38. Cha DJ, Franklin JL, Dou Y, Liu Q, Higginbotham JN, et al. 2015. KRASdependent sorting of miRNA to exosomes. eLife 4:eo7197

39. Janas T, Janas MM, Sapon K, Janas T. 2015. Mechanisms of RNA loading into exosomes. FEBS Lett. 589:1391-98

40. Ge Q, Zhou Y, Lu J, Bai Y, Xie X, Lu Z. 2014. miRNA in plasma exosome is stable under different storage conditions. Molecules 19:1568-75

41. Lotvall J, Hill AF, Hochberg F, Buzas EI, Di Vizio D, et al. 2014. Minimal experimental requirements for definition of extracellular vesicles and their functions: a position statement from the International Society for Extracellular Vesicles. J. Extracell. Vesicles 3:26913 
42. Oksvold MP, Kullmann A, Forfang L, Kierulf B, Li M, et al. 2014. Expression of B-cell surface antigens in subpopulations of exosomes released from B-cell lymphoma cells. Clin. Ther. 36:847-62.e1

43. Horejsi V, Vlcek C. 1991. Novel structurally distinct family of leucocyte surface glycoproteins including CD9, CD37, CD53 and CD63. FEBS Lett. 288:1-4

44. Moreno-Gonzalo O, Villarroya-Beltri C, Sa'nchez-Madrid F. 2014. Posttranslational modifications of exosomal proteins. Front. Immunol. 5:383

45. Sterzenbach U, Putz U, Low LH, Silke J, Tan SS, Howitt J. 2017. Engineered exosomes as vehicles for biologically active proteins. Mol. Ther. 25:1269-78

46. Subra C, Laulagnier K, Perret B, Record M. 2007. Exosome lipidomics unravels lipid sorting at the level of multivesicular bodies. Biochimie 89:205-12

47. Laulagnier K, Grand D, Dujardin A, Hamdi S, Vincent-Schneider H, et al. 2004. PLD2 is enriched on exosomes and its activity is correlated to the release of exosomes. FEBS Lett. 572:11-14

48. Laulagnier K, Motta C, Hamdi S, Roy S, Fauvelle F, et al. 2004. Mast cell- and dendritic cell-derived exosomes display a specific lipid composition and an unusual membrane organization. Biochem. J. 380:161-71

49. Takahashi A, Okada R, Nagao K, Kawamata Y, Hanyu A, et al. 2017. Exosomes maintain cellular homeostasis by excreting harmful DNA from cells. Nat. Commun. 8:15287

50. Kahlert C, Melo SA, Protopopov A, Tang J, Seth S, et al. 2014. Identification of double-stranded genomic DNA spanning all chromosomes with mutated KRAS and p53 DNA in the serum exosomes of patients with pancreatic cancer. $J$. Biol. Chem. 289:3869-75

51. San Lucas FA, Allenson K, Bernard V, Castillo J, Kim DU, et al. 2016. Minimally invasive genomic and transcriptomic profiling of visceral cancers by nextgeneration sequencing of circulating exosomes. Ann. Oncol. 27:635-41

52. Thakur BK, Zhang H, Becker A, Matei I, Huang Y, et al. 2014. Double-stranded DNA in exosomes: a novel biomarker in cancer detection. Cell Res. 24:766-69

53. Sansone P, Savini C, Kurelac I, Chang Q, Amato LB, et al. 2017. Packaging and transfer of mitochondrial DNA via exosomes regulate escape from dormancy in hormonal therapy-resistant breast cancer. PNAS 114:E9066-E75

54. Escrevente C, Grammel N, Kandzia S, Zeiser J, Tranfield EM, et al. 2013. Sialoglycoproteins and $\mathrm{N}$-glycans from secreted exosomes of ovarian carcinoma cells. PLOS ONE 8:e78631

55. Feng M, Chen JY, Weissman-Tsukamoto R, Volkmer JP, Ho PY, et al. 2015. Macrophages eat cancer cells using their own calreticulin as a guide: roles of TLR and Btk. PNAS 112:2145-50

56. Purushothaman A, Bandari SK, Liu J, Mobley JA, Brown EE, Sanderson RD. 2016. Fibronectin on the surface of myeloma cell-derived exosomes mediates exosome-cell interactions. J. Biol. Chem. 291:1652-63

57. Muller L, Mitsuhashi M, Simms P, Gooding WE, Whiteside TL. 2016. Tumorderived exosomes regulate expression of immune function-related genes in human T cell subsets. Sci. Rep. 6:20254 
58. Schirle NT, MacRae IJ. 2012. The crystal structure of human Argonaute2. Science 336:1037-40

59. Schirle NT, Sheu-Gruttadauria J, MacRae IJ. 2014. Structural basis for microRNA targeting. Science346:608-13

6o. Friedman RC, Farh KK, Burge CB, Bartel DP. 2009. Most mammalian mRNAs are conserved targets of microRNAs. Genome Res. 19:92-105

61. Zhou H, Rigoutsos I. 2014. MiR-103a-3p targets the $5^{\times}$UTR of GPRC5A in pancreatic cells. RNA 20:1431-39

62. Xu W, San Lucas A, Wang Z, Liu Y. 2014. Identifying microRNA targets in different gene regions. BMC Bioinform. 15(Suppl. 7):S4

63. Martinez J, Tuschl T. 2004. RISC is a $5^{\times}$phosphomonoester-producing RNA endonuclease. Genes Dev. 18:975-80

64. Fabbri M, Paone A, Calore F, Galli R, Gaudio E, et al. 2012. MicroRNAs bind to Toll-like receptors to induce prometastatic inflammatory response. PNAS 109:E2110-E16

65. Doan T, Melvold R, Viselli S, Waltenbaugh C. 2008. Immunology. Philadelphia, PA: Wolters Kluwer Health/Lippincott Williams \& Wilkins

66. Harrington JM, Shannon HS. 1974. Letter: Epidemic of aortic aneurysm? Lancet 2:1575

67. Sabroe I, Dower SK, Whyte MK. 2005. The role of Toll-like receptors in the regulation of neutrophil migration, activation, and apoptosis. Clin. Infect. Dis. 41(Suppl. 7):S421-S26

68. Sallusto F, Lanzavecchia A. 2002. The instructive role of dendritic cells on T-cell responses. Arthritis Res. Ther. 4(Suppl. 3):S127-S32

69. Yarovinsky F, Zhang D, Andersen JF, Bannenberg GL, Serhan CN, et al. 2005. TLR11 activation of dendritic cells by a protozoan profilin-like protein. Science 308:1626-29

70. Pifer R, Benson A, Sturge CR, Yarovinsky F. 2011. UNC93B1 is essential for TLR11 activation and IL-12-dependent host resistance to Toxoplasma gondii. J. Biol. Chem. 286:3307-14

71. Koblansky AA, Jankovic D, Oh H, Hieny S, Sungnak W, et al. 2013. Recognition of profilin by Toll-like receptor 12 is critical for host resistance to Toxoplasma gondii. Immunity 38:119-30

72. Mishra BB, Gundra UM, Teale JM. 2008. Expression and distribution of Toll-like receptors 11-13 in the brain during murine neurocysticercosis. $J$. Neuroinflamm. 5:53

73. Shi Z, Cai Z, Sanchez A, Zhang T, Wen S, et al. 2011. A novel Toll-like receptor that recognizes vesicular stomatitis virus. J. Biol. Chem. 286:4517-24

74. Oldenburg M, Kruger A, Ferstl R, Kaufmann A, Nees G, et al. 2012. TLR13 recognizes bacterial 23S rRNA devoid of erythromycin resistance-forming modification. Science 337:1111-15

75. Tatematsu M, Nishikawa F, Seya T, Matsumoto M. 2013. Toll-like receptor 3 recognizes incomplete stem structures in single-stranded viral RNA. Nat. Commun. 4:1833 
76. Forsbach A, Samulowitz U, Volp K, Hofmann HP, Noll B, et al. 2011. Dual or triple activation of TLR7, TLR8, and/or TLR9 by single-stranded oligoribonucleotides. Nucleic Acid Ther. 21:423-36

77. Eigenbrod T, Dalpke AH. 2015. Bacterial RNA: an underestimated stimulus for innate immune responses. J. Immunol. 195:411-18

78. Magini D, Giovani C, Mangiavacchi S, Maccari S, Cecchi R, et al. 2016. Selfamplifying mRNA vaccines expressing multiple conserved influenza antigens confer protection against homologous and heterosubtypic viral challenge. PLOS ONE 11:e0161193

79. Rivera J, Cordero RJ, Nakouzi AS, Frases S, Nicola A, Casadevall A. 2010. Bacillus anthracis produces membrane-derived vesicles containing biologically active toxins. PNAS 107:19002-7

8o. Lee J, Lee EY, Kim SH, Kim DK, Park KS, et al. 2013. Staphylococcus aureus extracellular vesicles carry biologically active $\beta$-lactamase. Antimicrob. Agents Chemother. 57:2589-95

81. Silverman JM, Clos J, de'Oliveira CC, Shirvani O, Fang Y, et al. 2010. An exosome-based secretion pathway is responsible for protein export from Leishmania and communication with macrophages. J. Cell Sci. 123:842-52

82. Meckes DG Jr. 2015. Exosomal communication goes viral. J. Virol. 89:5200-3

83. Zhang L, Hou D, Chen X, Li D, Zhu L, et al. 2012. Exogenous plant MIR168a specifically targets mammalian LDLRAP1: evidence of cross-kingdom regulation by microRNA. Cell Res. 22:107-26

84. Shu J, Chiang K, Zempleni J, Cui J. 2015. Computational characterization of exogenous microRNAs that can be transferred into human circulation. PLOS ONE 10:e0140587

85. Wang L, Sadri M, Giraud D, Zempleni J. 2018. RNase H2-dependent polymerase chain reaction and elimination of confounders in sample collection, storage, and analysis strengthen evidence that microRNAs in bovine milk are bioavailable in humans. J. Nutr. 148:153-59

86. Wolf T, Baier SR, Zempleni J. 2015. The intestinal transport of bovine milk exosomes is mediated by endocytosis in human colon carcinoma caco-2 cells and rat small intestinal IEC-6 cells. J. Nutr. 145:2201-6

87. Izumi H, Tsuda M, Sato Y, Kosaka N, Ochiya T, et al. 2015. Bovine milk exosomes contain microRNA and mRNA and are taken up by human macrophages. J. Dairy Sci. 98:2920-33

88. Kusuma Jati R, Manca S, Friemel T, Sukreet S, Nguyen C, Zempleni J. 2016. Human vascular endothelial cells transport foreign exosomes from cow's milk by endocytosis. Am. J. Physiol. Cell Physiol. 310:C80o-C7

89. Sukreet S, Zhang H, Adamec J, Cui J, Zempleni J. 2017. Identification of glycoproteins on the surface of bovine milk exosomes and intestinal cells that facilitate exosome uptake in human colon carcinoma Caco-2 cells. FASEB J. 31:646.25

9o. Krogh A, Larsson B, von Heijne G, Sonnhammer ELL. 2001. Predicting transmembrane protein topology with a hidden Markov model: application to complete genomes. J. Mol. Biol. 305(3):567-80 
91. Julenius K. 2007. NetCGlyc 1.0: prediction of mammalian C-mannosylation sites. Glycobiology 17:868-76

Gupta R, Jiung E, Brunak S. 2004. NetNGlyc 1.o Server. Lyngby, Den.: Tech. Univ. Den., accessed May 1, 2018. http://www.cbs.dtu.dk/services/NetNGlyc/

93. Steentoft C, Vakhrushev SY, Joshi HJ, Kong Y, Vester-Christensen MB, et al. 2013. Precision mapping of the human $O$-GalNAc glycoproteome through SimpleCell technology. EMBO J. 32:1478-88

94. Takov K, Yellon DM, Davidson SM. 2017. Confounding factors in vesicle uptake studies using fluorescent lipophilic membrane dyes. J. Extracell. Vesicles 6:1388731

95. Manca S, Upadhyaya B, Mutai E, Desaulniers AT, Cederberg RA, et al. 2018. Milk exosomes are bioavailable and distinct microRNA cargos have unique tissue distribution patterns. Sci. Rep. 8:11321

96. Munagala R, Aqil F, Jeyabalan J, Gupta RC. 2016. Bovine milk-derived exosomes for drug delivery. Cancer Lett. 371:48-61

97. Imai T, Takahashi Y, Nishikawa M, Kato K, Morishita M, et al. 2015. Macrophage-dependent clearance of systemically administered B16BL6derived exosomes from the blood circulation in mice. J. Extracell. Vesicles 4:26238

98. Chen X, Gao C, Li H, Huang L, Sun Q, et al. 2010. Identification and characterization of microRNAs in raw milk during different periods of lactation, commercial fluid, and powdered milk products. Cell Res. 20:1128-37

99. Izumi H, Kosaka N, Shimizu T, Sekine K, Ochiya T, Takase M. 2012. Bovine milk contains microRNA and messenger RNA that are stable under degradative conditions. J. Dairy Sci. 95:4831-41

100. Sun J, Aswath K, Schroeder SG, Lippolis JD, Reinhardt TA, Sonstegard TS. 2015. MicroRNA expression profiles of bovine milk exosomes in response to Staphylococcus aureus infection. BMC Genom. 16:806

101. Cai M, He H, Jia X, Chen S, Wang J, et al. 2018. Genome-wide microRNA profiling of bovine milkderived exosomes infected with Staphylococcus aureus. Cell Stress Chaperones 23:663-72

102. Zhou Q, Li M, Wang X, Li Q, Wang T, et al. 2012. Immune-related microRNAs are abundant in breast milk exosomes. Int. J. Biol. Sci. 8:118-23

103. Chen T, Xi QY, Ye RS, Cheng X, Qi QE, et al. 2014. Exploration of microRNAs in porcine milk exosomes. BMC Genom. 15:100

104. Benmoussa A, Lee CH, Laffont B, Savard P, Laugier J, et al. 2016. Commercial dairy cow milk microRNAs resist digestion under simulated gastrointestinal tract conditions. J. Nutr. 146:2206-15

105. Howard KM, Jati Kusuma R, Baier SR, Friemel T, Markham L, et al. 2015. Loss of miRNAs during processing and storage of cow's (Bos taurus) milk. J. Agric. Food Chem. 63:588-92

106. Ebert MS, Neilson JR, Sharp PA. 2007. MicroRNA sponges: competitive inhibitors of small RNAs in mammalian cells. Nat. Methods 4:721-26 
107. Chen T, Xie MY, Sun JJ, Ye RS, Cheng X, et al. 2016. Porcine milk-derived exosomes promote proliferation of intestinal epithelial cells. Sci. Rep. 6:33862

108. Cavalieri D, Rizzetto L, Tocci N, Rivero D, Asquini E, et al. 2016. Plant microRNAs as novel immunomodulatory agents. Sci. Rep. 6:25761

109. Wang K, Li H, Yuan Y, Etheridge A, Zhou Y, et al. 2012. The complex exogenous RNA spectra in human plasma: An interface with human gut biota? PLOS ONE 7:e51009

110. Ju S, Mu J, Dokland T, Zhuang X, Wang Q, et al. 2013. Grape exosome-like nanoparticles induce intestinal stem cells and protect mice from DSS-induced colitis. Mol. Ther. 21:1345-57

111. Mu J, Zhuang X, Wang Q, Jiang H, Deng ZB, et al. 2014. Interspecies communication between plant and mouse gut host cells through edible plant derived exosome-like nanoparticles. Mol. Nutr. Food Res. 58:1561-73

112. Lukasik A, Zielenkiewicz P. 2014. In silico identification of plant miRNAs in mammalian breast milk exosomes-a small step forward? PLOS ONE 9:e99963

113. Beatty M, Guduric-Fuchs J, Brown E, Bridgett S, Chakravarthy U, et al. 2014. Small RNAs from plants, bacteria and fungi within the order Hypocreales are ubiquitous in human plasma. BMC Genom. 15:933

114. Liang G, Zhu Y, Sun B, Shao Y, Jing A, et al. 2014. Assessing the survival of exogenous plant microRNA in mice. Food Sci. Nutr. 2:380-88

115. Zhou Z, Li X, Liu J, Dong L, Chen Q, et al. 2015. Honeysuckle-encoded atypical microRNA2911 directly targets influenza A viruses. Cell Res. 25:39-49

116. Yang J, Farmer LM, Agyekum AA, Hirschi KD. 2015. Detection of dietary plant-based small RNAs in animals. Cell Res. 25:517-20

117. Mlotshwa S, Pruss GJ, MacArthur JL, Endres MW, Davis C, et al. 2015. A novel chemopreventive strategy based on therapeutic microRNAs produced in plants. Cell Res. 25:521-24

118. Chin AR, Fong MY, Somlo G, Wu J, Swiderski P, et al. 2016. Cross-kingdom inhibition of breast cancer growth by plant miR159. Cell Res. 26:217-28

119. Chen X, Dai GH, Ren ZM, Tong YL, Yang F, Zhu YQ. 2016. Identification of dietetically absorbed rapeseed (Brassica campestris L.) bee pollen microRNAs in serum of mice. Biomed. Res. Int. 2016:5413849

120. Luo Y, Wang P, Wang X, Wang Y, Mu Z, et al. 2017. Detection of dietetically absorbed maize-derived microRNAs in pigs. Sci. Rep. 7:645

121. Cui J, Zhou B, Ross SA, Zempleni J. 2017. Nutrition, microRNAs, and human health. Adv. Nutr. 8:105- 12

122. Laubier J, Castille J, Le Guillou S, Le Provost F. 2015. No effect of an elevated miR-3ob level in mouse milk on its level in pup tissues. RNA Biol. 12:26-29

123. Auerbach A, Vyas G, Li A, Halushka M, Witwer K. 2016. Uptake of dietary milk miRNAs by adult humans: a validation study. F10ooRes. 5:721 
124. Title AC, Denzler R, Stoffel M. 2015. Uptake and function studies of maternal milk-derived microRNAs. J. Biol. Chem. 290:23680-91

125. Kang W, Bang-Berthelsen CH, Holm A, Houben AJ, Muller AH, et al. 2017. Survey of $800+$ data sets from human tissue and body fluid reveals xenomiRs are likely artifacts. RNA 23:433-45

126. Pond SM, Tozer TN. 1984. First-pass elimination: basic concepts and clinical consequences. Clin. Pharmacokinet. 9:1-25

127. Zempleni J, Baier SR, Hirschi K. 2015. Diet-responsive microRNAs are likely exogenous. J. Biol. Chem. 290:25197

128. Snow JW, Hale AE, Isaacs SK, Baggish AL, Chan SY. 2013. Ineffective delivery of diet-derived microRNAs to recipient animal organisms. RNA Biol. 10:1107-16

129. Ameres SL, Horwich MD, Hung JH, Xu J, Ghildiyal M, et al. 2010. Target RNAdirected trimming and tailing of small silencing RNAs. Science 328:1534-39

130. Ruegger S, Grosshans H. 2012. MicroRNA turnover: when, how, and why. Trends Biochem. Sci. 37:436- 46

131. Zempleni J. 2017. Milk exosomes: beyond dietary microRNAs. Genes Nutr. 12:12

132. Askenase P. 2018. Exosomes are the elephant in the room; their carrier effects likely influence target cells responses to miRNA transfers. eLife. In press

133. Guo Z, Karunatilaka KS, Rueda D. 2009. Single-molecule analysis of proteinfree U2-U6 snRNAs. Nat. Struct. Mol. Biol. 16:1154-59

134. Maden BE, Hughes JM. 1997. Eukaryotic ribosomal RNA: the recent excitement in the nucleotide modification problem. Chromosoma 105:391-400

135. Reeves PG, Nielsen FH, Fahey GC Jr. 1993. AIN-93 purified diets for laboratory rodents: final report of the American Institute of Nutrition ad hoc writing committee on the reformulation of the AIN-76A rodent diet. J. Nutr. 123:1939-51

136. Ward RE, Ninonuevo M, Mills DA, Lebrilla CB, German JB. 2006. In vitro fermentation of breast milk oligosaccharides by Bifidobacterium infantis and Lactobacillus gasseri. Appl. Environ. Microbiol. 72:4497-99

137. Mutai E, Zhou F, Zempleni J. 2017. Depletion of dietary bovine milk exosomes impairs sensorimotor gating and spatial learning in C57BL/6 mice. FASEB J. 31:150.4

138. Duster R, Prickaerts J, Blokland A. 2014. Purinergic signaling and hippocampal long-term potentiation. Curr. Neuropharmacol. 12:37-43

139. Aguilar-Lozano A, Baier S, Grove R, Shu J, Giraud D, et al. 2018. Concentrations of purine metabolites are elevated in fluids from adults and infants and in livers from mice fed diets depleted of bovine milk exosomes and their RNA cargos. J. Nutr. 148(12):1886-94 
140. Leiferman A, Shu J, Grove R, Cui J, Adamec J, Zempleni J. 2018. A diet defined by its content of bovine milk exosomes and their RNA cargos has moderate effects on gene expression, amino acid profiles and grip strength in skeletal muscle in C57BL/6 mice. J. Nutr. Biochem. 59:123-28

141. Vilella F, Moreno-Moya JM, Balaguer N, Grasso A, Herrero M, et al. 2015. Hsa-miR-3od, secreted by the human endometrium, is taken up by the preimplantation embryo and might modify its transcriptome. Development 142:3210-21

142. Sadri M, Xie F, Wood J, Zempleni J. 2016. Dietary depletion of cow's milk microRNAs impairs fecundity in mice. FASEB J. 30:673.5

143. Panwala CM, Jones JC, Viney JL. 1998. A novel model of inflammatory bowel disease: mice deficient for the multiple drug resistance gene, mdria, spontaneously develop colitis. J. Immunol. 161:5733-44

144. Agrawal AK, Aqil F, Jeyabalan J, Spencer WA, Beck J, et al. 2017. Milk-derived exosomes for oral delivery of paclitaxel. Nanomedicine 13:1627-36 